\title{
ON THE BIOSYNTHESIS OF RIBOFLAVIN IN THE CULTURE OF EREMOTHECIUM ASHBYII
}

\author{
SATORU KUWADA, TORU MASUDA, TOYOKAZU KISHI \\ AND MITSUKO ASAI \\ Research Laboratories, Takeda Pharmaceutical Industries, Ltd. ${ }^{2}$
}

(Received June 16, 1958)

Masuda (1-2), one of the authors, previously isolated from the mycelium of Eremothecium ashbyii a green fluorescent substance, G Compound, and a purple fluorescent substance, V Compound, along with riboflavin, flavin-adenine dinucleotide and others, and assumed the former to be 2,4-dioxo-6,7-dimethyl8-ribitylpteridine $(1,3)$ and the latter 2,4-dioxo-6-methyl-7-hydroxy-8-ribitylpteridine (2, 4-5). Thereafter, the authors decided to call their mother nucleus (2,4-dioxo-8-ribitylpteridine) Ribolumazine (6) and hence to call the two compounds 6,7-dimethyl-ribolumazine and 6-methyl-7-hydroxy-ribolumazine, respectively, because the names $\mathrm{G}$ and $\mathrm{V}$ Compounds may cause confusion as they are also used for other compounds and because their full chemical names are too long.

Masuda (3) formerly presumed 6,7-dimethyl-ribolumazine to be an intermediate in the biosynthesis of riboflavin and demonstrated that riboflavin could be readily produced by the reaction of the compound with diacetyl or with acetoin in vitro.

Thereafter, Katagiri (7) biosynthesized riboflavin from the 6,7-dimethylribolumazine sent by the authors, using a cell-free extract of Escherichia coli or resting cells of acetone-butanol-producing bacterium (Clostridium acetobutyricum) and announced that the addition of pyruvic acid in that case increased the formation of riboflavin. He also reported at the 101 st and $102 \mathrm{nd}$ Meetings of the Vitamin B Research Committee that the above biosynthesis could also be effected by using a solution of the enzyme of Er. ashbyii.

From these results it may be said that the authors' assumption for the mechanism of the biosynthesis of riboflavin has been materialized in part. As the biosynthesis of riboflavin from 6,7-dimethyl-ribolumazine has been evidenced, the remaining problem is to know how the lumazine derivative is biosynthesized. As will be described in the part of Discussion, the isoalloxazine nucleus of riboflavin may be formed from various factors such as purine, pyrimidine, amino acid, etc., but the authors have reached the conclusion that it is most reasonable to think that riboflavin is biosynthesized

\footnotetext{
1 The summary of this work was presented at the Symposium of the Vitamin B Research Committee (Feb. 28, 1958).

2 Juso-nishino-cho, Higashiyodogawa-ku, Osaka.
} 
by a series of reactions such as purine compound $\rightarrow$ uracil compound $\rightarrow$ lumazine compound $\rightarrow$ riboflavin.

In regard to the conversion in vivo of a purine compound to an uracil compound, there remain many problems to be discussed, and the authors have also so far been unable to confirm the conversion experimentally. For example, the reaction of 6,7-dimethyl-lumazine with diacetyl or acetoin in vitro did not produce lumichrome, but the same reaction with 6,7,8-trimethyllumazine yielded lumiflavin. This fact shows that the reactivity of the methyl groups at C-6 and C-7 is considerably different according to the presence or absence of a substituent at N-8. Based on this view-point, the authors attempted the cultivation of Er. ashbyii in the presence of 4,5-diaminouracil as a model experiment of the biosynthesis, but no derivative of lumazine or isoalloxazine could be detected in the broth and mycelium. The same experiment was further carried out in the presence of 4-methylamino-5-aminouracil, but though the formation of 6,7,8-trimethyl-lumazine was confirmed, lumiflavin failed to be detected. This clearly indicates that such a reaction gives a different result according to the condition whether it is conducted in vitro or in vivo. If the amino group at $\mathrm{C}-4$ of the diaminouracil possesses a ribityl group, the compound may enter the metabolic system to form the isoalloxazine nucleus. Based upon this assumption, various experiments are now under way and the meaning of 6-methyl-7-hydroxy-ribolumazine is also under investigation.

\section{EXPERIMENTAL}

1. 6,7-Dimethyl-ribolumazine ( $\mathrm{G}$ Compound, $\mathrm{C}_{13} \mathrm{H}_{18} \mathrm{O}_{6} \mathrm{~N}_{4}$ ).

Yellow needles, mp 273-274 (decomp.), obtained from the mycelium of Er. ashbyii by the method of Masuda (1).

2. 6-Methyl-7-hydroxy-ribolumazine (V Compound, $\mathrm{C}_{12} \mathrm{H}_{16} \mathrm{O}_{7} \mathrm{~N}_{4}$ ).

Colorless needles, mp $263^{\circ}$ (decomp.), obtained from the mycelium of $\mathrm{Er}$. ashbyii by the method of Masuda, Kishi, and Asai (2).

3. Reaction Between 6,7-Dimethyl-ribolumazine and Diacetyl or Acetoin.

Riboflavin was produced by the reaction between both compounds and identified by paper partition chromatography (PPC), ultraviolet spectrum and bioassay as reported by Masuda (3).

4. Reaction Between 6,7-Dimethyl-lumazine and Diacetyl or Acetoin.

To $5 \mathrm{mg}$ of 6,7-dimethyl-lumazine was added $1 \mathrm{ml}$ of diacetyl or acetoin and the mixture was heated at ca. $130^{\circ}$ for $4-5$ hours. After cooling, the reaction mixture was examined by PPC to give the results shown in Table I.

Table I contains also the results of the reactions conducted in the presence of $0.05 \mathrm{ml}$ of piperidine. In all cases, a blue-fluorescent spot failed to be detected at the site corresponding to the $R_{\mathrm{F}}$ of lumichrome.

5. Reaction Between 6,7,8-Trimethyl-lumazine and Diacetyl.

To $5 \mathrm{mg}$ of 6,7,8-trimethyl-lumazine synthesized by Masuda's method (8) was added $1 \mathrm{ml}$ of diacetyl and the mixture was refluxed at $120^{\circ}$ for 1.5 hours 
TABLE I

I'aper Chromalograms of the Reaction Products of Dimethyllumazine

I, acetic acid-butanol-water $(1: 4: 5)$. II, pyridine-butanol-water $(3: 4: 7)$

\begin{tabular}{|c|c|c|}
\hline \multirow{2}{*}{ Sample } & \multicolumn{2}{|c|}{$R_{\mathrm{F}}$ value using the solvent system } \\
\hline & I & II \\
\hline 6,7-Dimethyl-lumazine plus diacetyl & $0.44 \mathrm{~GB}^{a}$ & $0.71 \mathrm{~GB}$ \\
\hline " plus piperidine & 0.44 & 0.70 \\
\hline 6,7-Dimethyl-lumazine plus acetoin & 0.43 & $0.70 "$ \\
\hline$"$ plus piperidine & 0.44, & 0.71, \\
\hline 6,7-Dimethyl-lumazine & 0.44, & 0.71, \\
\hline Lumichrome & $0.57 \quad \mathrm{~B}^{b}$ & $0.76 \mathrm{~B}$ \\
\hline
\end{tabular}

${ }^{a} \mathrm{~GB}$, greenish-blue fluorescence.

${ }^{b} \mathrm{~B}$, blue fluorescence.

TABLE II

Paper Chromatograms of the Reaction Products of Trimethyllumazine

\begin{tabular}{|c|c|c|c|c|c|}
\hline \multirow[b]{2}{*}{ Sample } & \multicolumn{5}{|c|}{$R_{\mathrm{F}}$ value and color of fluorescence ${ }^{a}$} \\
\hline & $\mathrm{v}^{0.07} \pm$ & $\mathrm{G}^{0.22} \mathrm{III}$ & $\begin{array}{c}0.29 \\
?\end{array}$ & $\mathrm{Y}^{0.37} \mathrm{H}$ & $\begin{array}{c}0.43 \\
\mathrm{YR} H\end{array}$ \\
\hline Lumiflavin & & & & $\stackrel{0.38}{\mathrm{Y}}$ & \\
\hline
\end{tabular}

${ }^{a} \mathrm{~V}$, violet; G, green; Y, yellow; YR, yellowish-red fluorescence. \pm to $\mathrm{Hl}$ indicate the intensity of fluorescence. The same is applicable to the following tables.

in an oil bath. The reaction mixture was developed on a filter paper with acetic acid-butanol-water $(1: 4: 5)$ and the spot corresponding to $R_{\mathrm{F}} 0.37$, detected by ultraviolet light, was found to be in accord with that of lumiflavin (Table II).

The same experiment was repeated using a larger sheet of filter paper and the fluorescent spot corresponding to $R_{\mathrm{F}} 0.37$ was cut out and extracted with alcohol. The extract gave the same ultraviolet absorption spectrum as that of the lumiflavin prepared from riboflavin.

6. Action of 4,5-Diaminouracil in the Culture of Er. ashbyii.

To $500 \mathrm{ml}$ of a medium consisting, in per cent, of polypeptone, 0.8 ; bonito extract, $0.8 ; \mathrm{MgSO}_{4} \cdot 7 \mathrm{H}_{2} \mathrm{O}, 0.01 ; \mathrm{KH}_{2} \mathrm{PO}_{4}, 0.2 ; \mathrm{NaCl}, 0.1$ and glucose, 2.0, was added 200 or $400 \mathrm{mg}$ of 4,5-diaminouracil, and Er. ashbyii was cultivated in the medium for 4 days with shaking. The mycelium separated by filtration was extracted with water at $80^{\circ}$ and the extract was concentrated in vacuo. The broth was also concentrated under reduced pressure. Both samples thus obtained were subjected to PPC and examined with ultraviolet light, respectively, but no fluorescent spot was detected at the site corresponding to the $R_{\mathrm{F}}$ of 6,7-dimethyl-lumazine or lumichrome. As shown in Table III, where the amounts of the mycelium and resulting riboflavin are listed, the presence of 4,5-diaminouracil inhibited the biosynthesis of riboflavin. 
TABLE III

Amount of Riboflavin in the Culture of Er. ashbyii

\begin{tabular}{|c|c|c|c|c|}
\hline \multirow{2}{*}{ Culture medium } & \multirow{2}{*}{ Amount of mycelium } & \multicolumn{3}{|c|}{ Amount of riboflavin in } \\
\hline & & Mycelium & Broth & Mycelium plus broth \\
\hline \multirow{3}{*}{$\begin{array}{l}\text { Basal medium } \\
\text { Basal medium plus } \\
4,5 \text {-diaminouracil }\end{array}$} & $\mathrm{g} / 100 \mathrm{ml}$ & $m g / g$ & $m g / 100 m l$ & $m g / 100 \mathrm{ml}$ \\
\hline & 2.67 & 5.06 & 13.2 & 26.7 \\
\hline & 2.38 & 3.4 & 9.0 & 17.1 \\
\hline
\end{tabular}

7. Biosynthesis of 6,7,8-Trimethyl-lumazine from 4-Methylamino-5-aminouracil.

To $500 \mathrm{ml}$ of the same medium as in 6 was added $50 \mathrm{mg}$ of 4-methylamino5-aminouracil and Er. ashbyii was cultivated in the medium for 3 days with shaking. The broth was concentrated to about $50 \mathrm{ml}$ in vacuo. On the other hand, $5 \mathrm{~g}$ of the mycelium was extracted with hot water and the extract was also concentrated to ca. $5 \mathrm{ml}$. The two samples were developed on a filter paper with acetic acid-butanol-water (1:4:5) and examined with ultraviolet light to give the results shown in Table IV.

TABLE IV

Paper Chromatograms of the Culture of Er. ashbyii

\begin{tabular}{|c|c|c|c|c|c|}
\hline Substance & \multicolumn{5}{|c|}{$R_{\mathrm{F}}$ value and color of fluorescence } \\
\hline $\begin{array}{l}\text { Mycelium in the normal } \\
\text { culture }\end{array}$ & $0.03 \mathrm{Y}$ & $0.13 \mathrm{G}$ & $0.15 \mathrm{~V}$ & & $0.28 \mathrm{Y}$ \\
\hline $\begin{array}{l}\text { Mycelium in the culture in } \\
\text { the presence of } 4 \text {-methyl- } \\
\text { amino-5-amino uracil }\end{array}$ & $0.01 \mathrm{Y}$ & $0.13 \mathrm{G}$ & $0.15 \mathrm{~V}$ & $0.25 \mathrm{G}($ (?) & $0.28 \mathrm{Y}$ \\
\hline $\begin{array}{l}\text { Broth in the above culture } \\
6,7,8 \text {-trimethyl-lumazine }\end{array}$ & $0.01 \mathrm{Y}$ & $0.13 \mathrm{G}$ & $0.15 \mathrm{~V}$ & $\begin{array}{l}0.26 \mathrm{G} \\
0.26 \mathrm{G}\end{array}$ & $0.28 \mathrm{Y}$ \\
\hline & $\stackrel{\uparrow}{\mathrm{FAD}}$ & $\begin{array}{l}\uparrow \\
\text { 6,7-Dimethyl- } \\
\text { ribolumazine }\end{array}$ & $\begin{array}{c}\uparrow \\
\text { 6-Methyl } \\
\text { hydroxy- } \\
\text { riboluma }\end{array}$ & & $\uparrow_{\text {Riboflavin }}$ \\
\hline
\end{tabular}

As seen from the Table the green fluorescent substance with $R_{\mathrm{F}} 0.26$ failed to be detected in the extract of the mycelium obtained by the culture in the basal medium alone. The fluorescent substance was identified as 6,7,8-trimethyl-lumazine because the $R_{\mathrm{F}}$ value and color of the fluorescence of the substance were in complete agreement with those of the same compound chemically synthesized by Masuda's method (8). From this result, it was learned that the compound could be biosynthesized from 4-methylamino-5-amino-uracil by Er. ashbyii. In this case, however, no spot recognizable as lumiflavin was detected.

\section{DISCUSSION}

Many reviews (9) have so far been published on the biosynthesis of riboflavin, but here are cited only those which have close relation with the authors' 
studies.

MacLaren (10) is the first who advanced the view that purine compounds would play the role of precursor in the biosynthesis of riboflavin. Giri and Krishnaswamy (11) observed that in the formation of riboflavin by a mutant of Saccharomyces cerevisiae, any of purine, pyrimidine and amino acids would facilitate the biosynthesis. On the other hand, McNutt (12), having found that free purine compounds are suitable for riboflavinogenesis by Er. ashbyii, attempted the biosynthesis of riboflavin from adenine-8-C $\mathrm{C}^{14}$ in order to make clear the process of conversion of the purine nucleus into the riboflavin nucleus, but as the resulting riboflavin and its photolytic product, lumichrome, had no radioactivity, he supposed that C-8 of adenine did not enter the riboflavin nucleus. He also produced riboflavin from randomly labelled $\mathrm{C}^{14}$-adenine and after investigating the radioactivity of the photolytic product found that 80 per cent of the $\mathrm{C}^{14}$ entered the isoalloxazine nucleus. The presumption was derived from this result that the isoalloxazine nucleus would be formed by the combination of diaminopyrimidine, which is a degradation product of purine, or its ribityl derivative with a proper aliphatic compound. Also, McNutt (13) conducted the same experiment using uniformly labelled $\mathrm{C}^{14}$-adenine and after investigating the resulting $\mathrm{C}^{14}$-riboflavin and its photolytic product, observed that 94 per cent of the $\mathrm{C}^{14}$ of the former entered the pyrimidine nucleus of the latter. As correlation between $\mathrm{C}^{14}$ 's of the nucleus in radioactivity $w$ as the same as in the original $\mathrm{C}^{14}$-adenine, he assumed that adenine had been consumed as such for the formation of the pyrimidine nucleus of riboflavin.

Klungsöyr (14) incubated the mycelium of Er. ashbyii in an inorganic salt buffer in the presence of $\mathrm{CH}_{3} \mathrm{C}^{14} \mathrm{OOH}$ and $\mathrm{HC}{ }^{14} \mathrm{OOH}$ to know whether $\mathrm{C}^{14}$ entered riboflavin. However, while radioactivity was detected in the lumiflavin and lumichrome produced by photolysis of the resulting riboflavin, no activity was found in 1,2-dihydro-1,6,7-trimethyl-2-keto-3-quinoxalinecarboxylic acid prepared by the hydrolysis of lumiflavin. He thought it therefore to be certain that the $\mathrm{C}^{14}$ of $\mathrm{CH}_{3} \mathrm{C}^{14} \mathrm{OOH}$ and $\mathrm{HC}^{14} \mathrm{OOH}$ entered the riboflavin as $\mathrm{C}-2$.

Goodwin and Pendlington (15) considered that of amino acids L-threonine, L-serine and L-tyrosine acted favorably for the biosynthesis of riboflavin by Er. ashbyii and that though purine, xanthine, adenine and adenosine were also suitable for the biosynthesis, pyrimidine had no such activity. Brown, Goodwin and Pendlington (16) stated afterwards that in the biosynthesis of riboflavin by Er. ashbyii only pyruvate was favorable among compounds containing no nitrogen, acetate was rather inhibitory, and further that 4,5-diaminouracil and other various pyrimidine compounds exerted no influence on the biosynthesis, but probably the ribityl derivative of 4,5-diaminouracil would be most effective as previously presumed by McNutt (12). Most recently, Good-

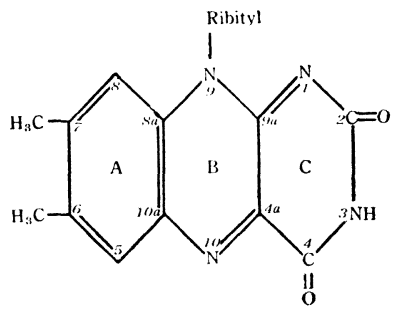

Fig. 1 Numbers of $C$ and $N$ in Riboflavin 
win and Treble (17) announced that lumazine, possibly produced from 4,5diaminouracil and glyoxal, could be detected when Er. ashbyii was incubated together with glyoxal. Goodwin and Johnes (18) duplicated the before-mentioned cultivation of Er. ashbyii in the presence of $\beta$-C $\mathrm{C}^{14}$-serine and found that the $\mathrm{C}^{14}$ entered riboflavin chiefly as $\mathrm{C}-2$ and that $\mathrm{C}-4 \mathrm{a}$ and $\mathrm{C}-9 \mathrm{a}$ showed only a little activity, whereas the $\mathrm{A}$ ring and ribityl side-chain exhibited no activity. This fact may be well explained by the before-mentioned Klungsöyr's theory (14), if serine is considered to be degraded into glycine and formate before entering the riboflavin nucleus. From this viewpoint, the supposition of Goodwin, et al. (15) that amino acids and purines enter as such into process of riboflavin synthesis becomes unreasonable. It seems rational to assume that amino acids are utilized for the formation of the pyrimidine nucleus through purines as suggested from Plaut's assertion (19) that the pyrimidine nucleus of pteridine and riboflavin is formed from purine compounds. Besides, Brown, Goodwin and Johnes (20) stated that various purine compounds contribute to the biosynthesis of riboflavin in different degrees, but it is impossible to draw a cogent conclusion from their theory.

Plaut (19) cultivated Ashbya gossypii in the presence of radioactive $\mathrm{C}_{2}^{*}$, II $\stackrel{*}{\mathrm{COOH}}, \mathrm{NH}_{2} \mathrm{CH}_{2} \stackrel{*}{\mathrm{COOOH}}$ to know the site of the active carbon (marked with $*$ ) in the resulting purine and riboflavin, and Weygand (21) applied the Plaut's results to the biosynthesis of leucopterine. Thereafter, Plaut (22) conducted the same cultivation in the presence of glucose-1- $\mathrm{C}^{14}$, glucose- $6-\mathrm{C}^{14}, \mathrm{CH}_{3}-\mathrm{C}^{14} \mathrm{OOH}$ and $\mathrm{C}^{14} \mathrm{H}_{3} \mathrm{COOH}$ and after investigating the radioactivity in the $\mathrm{A}$ ring of the resulting riboflavin, decided the site of $\mathrm{C}^{14}$ in the ring. Thus, the $\mathrm{A}$ ring of isoalloxazine seems to be formed from two-carbon precursors such as acetic acid, and the result of the experiments on lumichrome biosynthesis by Birch and Moye (23) appears to support this presumption.

When these reports are looked over, it becomes clear that the processes of the formation of purine, pteridine and riboflavin are all resembling, and the pyrimidine ring of purine seems to be utilized as such for the formation of the pyrimidine ring of pteridine or riboflavin. Therefore, Kato's assumption (24) that the isoalloxazine nucleus is formed in the order of the A, B and $C$ rings seems to be untenable. At any rate, as no definite intermediates have thus far been isolated in these biosyntheses, it cannot be denied that all the above views involve assumption to some extent.

6,7-Dimethyl-ribolumazine and 6-methyl-7-hydroxy-ribolumazine isolated by the authors from Er. ashbyii have a pteridine nucleus and a ribityl group, respectively, but lack the A ring of riboflavin. In addition, the former condenses with diacetyl or acetoin in vitro to give riboflavin (3). Since acetoin is contained in the mycelium and broth of Er. ashbyii in large quantities, the authors assumed the process of the biosynthesis of riboflavin by this microorganism to be as shown in the chart below.

This assumpion is in good accord with that of Plaut (22) but as acetic acid is reversible with diacetyl or acetion in vivo, any of them may be considered as the two-carbon precursor compound.

The presumption by many researchers that riboflavin is biosynthesized 


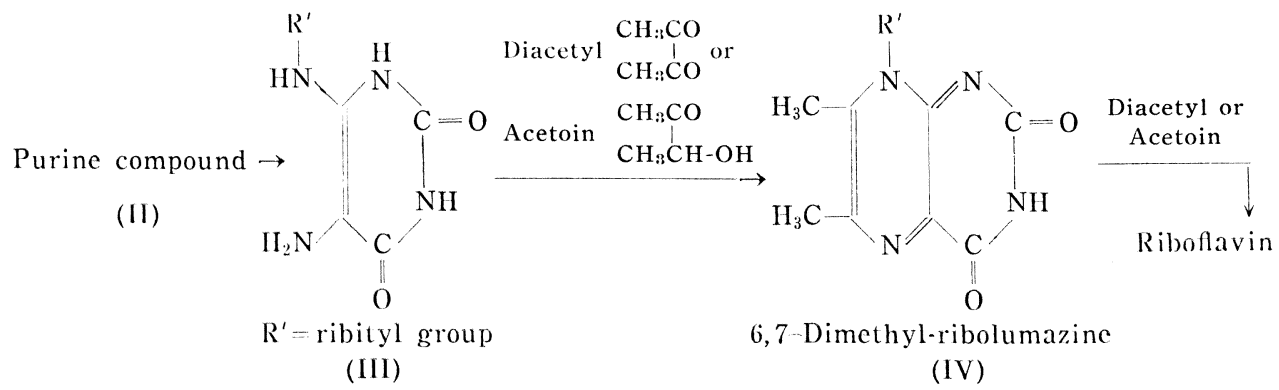

from purine compounds is well explained when 6,7-dimethyl-ribolumazine is considered as an intermediate in the synthesis. Albert (25) obtained 2-hydroxypteridine in 80 per cent yield by incubation of 2-hydroxypurine and glyoxal. Thereafter, he applied this reaction to glyoxalic acid, obtaining a pteridine compound in a good yield, but found that the same reaction with guanine or hypoxanthine was effected with difficulty. He also suggested the biochemical significance of this reaction. Formation of 6,7-dimethyl-ribolumazine by $\mathrm{Er}$. ashbyii discovered by the authors, can be explained by this view of Albert, and the assumption (12-13) that $\mathrm{C}-8$ of the purine nucleus is not consumed for the formation of riboflavin, is supported when 6,7-dimethyl-ribolumazine is considered to be an intermediate for the formation of riboflavin from purine.

Katagiri, et at. (7), attested the formation of riboflavin from 6,7-dimethylribolumazine, using Clostridium acetobutyricum and E. coli and found that the addition of pyruvic acid increased the formation, making clear that this ribolumazine is an intermediate in the biosynthesis of riboflavin.

Next, the authors supposed (4) the following machanism for the formation of 6-methyl-7-hydroxy-ribolumazine: The assumptive compound (III) condenses with diacetyl to give 6,7-dimethyl-ribolumazine, which in turn reacts with pyruvic acid to produce 6-methyl-7-hydroxy-ribolumazine. Although the biochemical significance of this compound is not yet clear, the above supposition seems to corroborate the existence of 4-ribitylamino-5-aminouracil (III) presumed by McNutt (12) and Goodwin, et al. (16).

In the cultivation of Er. ashbyii the authors found that the addition of 4,5-diaminouracil rather inhibited the formation of riboflavin. In this case,

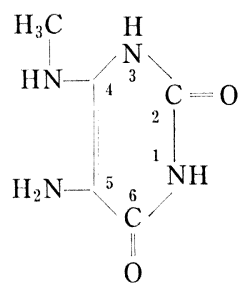

4-Methylamino-5-aminouracil

(VI)

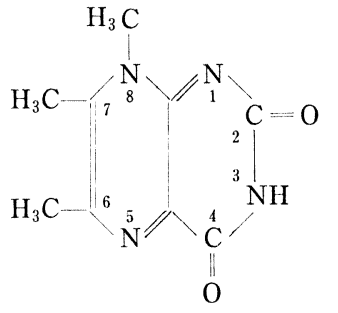

6,7,8-Trimethyl-lumazine (VII) 
the formation of dimethyl-lumazine or lumichrome by the reaction between the uracil compound and acetoin was also expected, but the formation could not be confirmed. On the other hand, when 4-methylamino-5-aminouracil (VI) was added instead of 4,5-diaminouracil, 6,7,8-trimethyl-lumazine (VIII) was biosynthesized.

From this fact, it seems certain that when the $\mathrm{NH}_{2}$-group at $\mathrm{C}-4$ of 4,5 diaminouracil has no substituent, the compound does not participate in the biosynthesis of pteridine compounds. The authors further presumed the biosynthesis of lumiflavin starting from the above biosynthesis, but the experiments failed against the anticipation. When 6,7,8-trimethyl-lumazine was allowed to react with diacetyl in a test tube, the formation of lumiflavin, though qualitatively, was proved. The mechanism appears the same as that in the formation of riboflavin by the reaction in vitro of 6,7-dimethyl-ribolumazine with diacetyl or acetoin. However, the fact that the trimethyl compound is formed in the broth of Er. ashbyii rather than lumiflavin, bespeaks that this microorganism does not utilize 6,7,8-trimethyl-lumazine as it does 6,7-dimethyl-ribolumazine, i.e., if the N-8 has no ribityl group, the compound is not utilized for further biosyntheses.

\section{SUMMARY}

First, mention was made that $\mathrm{G}$ Compound (2,4-dioxo-6,7-dimethyl-8-ribitylpteridine) and $\mathrm{V}$ Compound (2,4-dioxo-6-methyl-7-hydroxy-8-ribityl-pteridine), isolated from the culture product of Er. ashbyii, were renamed 6,7-dimethylribolumazine and 6-methyl-7-hydroxy-ribolumazine, respectively.

6, 7-Dimethyl-ribolumazine was assumed to be an intermediate in the biosynthesis of riboflavin on the basis of the results of chemical studies by Masuda and biochemical studies by Katagiri, et al. It has been recognized by many researchers that purine compounds play an important role in the biosynthesis of riboflavin. It has also been assumed that when a purine compound is added prior to the cultivation of Er. ashbyii, its C-8 is not utilized for the biosynthesis and that the pyrimidine nucleus enters as such in the isoalloxazine nucleus. So the authors investigated the relationship between 6,7-dimethyl-ribolumazine and purine compounds, especially uracil derivatives which are the degradation products of purines.

Formation of 6,7-dimethyl-ribolumazine is readily understood if it is considered that 4,5-diaminouracil, a compound lacking C-8 of xanthine, is first produced, and a ribityl group then enters $\mathrm{N}-4$ of the product to give 4-ribitylamino-5-aminouracil, which in turn reacts with diacetyl or with acetoin. Therefore, if such a uracil derivative is isolated from the culture products of Er. ashbyii, it is most powerful to prove the route of the biosynthesis, but in reality the formation of such a compound has not been confirmed.

According to the authors' studies, the addition of 4,5-diaminouracil prior to the cultivation of Er. ashbyi rather checked the formation of riboflavin and neither 6,7-dimethyl-lumazine nor lumichrome could be detected. When 4-methyl-amino-5-aminouracil instead of 4,5-diaminouracil was added, 6, 7,8- 
trimethyl-lumazine was found in the broth. In spite of the fact that this lumazine derivative readily reacts with diacetyl in vitro to give lumiflavin, no lumiflavin could be detected in the above broth. Hence, it should be considered that when a uracil derivative is converted to riboflavin via a lumazine compound, it is utilized for the biosynthesis only when its $\mathrm{NH}_{2}$ at $\mathrm{C}-4$ is substituted by a ribityl group.

\section{ACKNOWLEDGEMENT}

The authors wish to express their grateful thanks to Dr. Tsunaji Kusaka and Mr. Yutaka Shiraishi for their cooperations in the culture of Er. ashbyii.

\section{REFERENCES}

1. Masuda, T., Pharm. Bull. Japan 4, 375 (1956).

2. Masuda, T., Kishi, T. and Asai, M., ibid. 5, 598 (1957).

3. Masuda, T., ibid. 5, 136 (1957).

4. Masuda, T., Kishi, T. and Asai, M., Chem. Pharm. Bull. Japan 6, 113 (1958).

5. Masuda, T., Kishi, T. and Asai, M., ibid. 6, 291 (1958).

6. Kuwada, S., Masuda, T., Kishi, T. and Asai, M., ibid. 6, 447 (1958).

7. Katagiri, H., Imai, K., Takeda, I. and Yamada, H., Vitamins 12, 480 (1957).

8. Masuda, T., Pharm. Bull. Japan 5, 28 (1957).

9. Johnson, B. C., Ann. Rev. Biochem. 24, 431 (1955) ; Yagi, K. and Matsuoka, Y., Medical Science (Japan) 7, 110 (1955) ; Plaut, G. W. E. and Betheil, J. J, Ann. Rev. Biochem. 25, 479 (1956); Novelli, G. P., ibid. 26, 262 (1957).

10. MacLaren, J. A., J. Bacteriol. 63, 233 (1952).

11. Giri, K. V. and Krishnaswamy, P. R., ibid. 67, 309 (1954).

12. McNutt, W. S., J. Biol. Chem. 210, 511 (1954).

13. McNutt, W. S., ibid. 219, 365 (1956).

14. Klungsöyr, L. K., Acta Chim. Scand. 8, 723, 1292 (1954).

15. Goodwin, T. W. and Pendlington, S., Biochem. J. 57, 631 (1954).

16. Brown, E. G., Goodwin, T. W. and Pendlington, S., ibid. 61, 37 (1955).

17. Goodwin, T. W. and Treble, D. H., ibid. 67, 10p (1957).

18. Goodwin, T. W. and Jones, O. T. G., ibid. 64, 9 (1956).

19. Plaut, G. W. E., J. Biol. Chem. 208, 513 (1954).

20. Brown, E. G., Goodwin, T. W. and Jones, O. T. G., Biochem. J. 64, 37p (1956).

21. Weygand, F., Angew. Chem. 67, 328 (1955).

22. Plaut, G. W. E., J. Biol. Chem. 211, 111 (1954); Federation Proc. 13, 274 (1954).

23. Birch, A. J. and Moye, C. J., J. Chem. Soc. 1957, 412.

24. Kato, S., Vitamins 14, 50 (1958).

25. Albert, A., Biochem. J. 57, x (1954).

26. Albert, A., ibid. 65, 124 (1956). 